\title{
LMI1-like and KNOX1 genes coordinately regulate plant leaf development in dicotyledons
}

\author{
Lijing Chang ${ }^{1} \cdot$ Gaofu Mei ${ }^{1}$ Yan Hu ${ }^{1,2}$. Jieqiong Deng ${ }^{1}$ Tianzhen Zhang ${ }^{1,2}$
}

Received: 17 June 2018 / Accepted: 21 January 2019 / Published online: 28 January 2019

(c) The Author(s) 2019

\begin{abstract}
Key message This report reveals that the LMI1-like and KNOX1 genes coordinately control the leaf development and different combinations of those genes which produce diverse leaf shapes including broad, lobed and compound leaves. Abstract Class I KNOTTED1-like homeobox (KNOXI) genes are involved in compound leaf development and are repressed by the ASYMMETRIC LEAVES1 (AS1)-AS2 complex. Cotton plants have a variety of leaf shapes, including broad leaves and lobed leaves. GhOKRA, a LATE MERISTEM IDENTITY 1 (LMII)-like gene, controls the development of an okra leaf shape. We cloned the corresponding cotton homologs of Arabidopsis thaliana AS1 and AS2 and seven KNOXI genes. Through virus-induced gene silencing technology, we found that either GhASl or GhAS2-silenced cotton plants showed a great change in leaf shape from okra leaves to trifoliolate dissected leaves. In the shoot tips of these plants, the expression of the cotton ortholog of Knotted in A. thaliana 1 (KNAT1), GhKNOTTED1-LIKE2/3/4 (GhKNL2/3/4), was increased. However, GhKNOX1s-silenced plants maintained the wild-type okra leaves. A novel dissected-like leaf in A. thaliana was further generated by crossing plants constitutively expressing GhOKRA with either as 1-101 or as2-101 mutant plants. The dissected-like leaves showed two different leaf vein patterns. This report reveals that the LMII-like and KNOXI genes coordinately control leaf development, and different combinations of these genes produce diverse leaf shapes including broad leaves, lobed leaves and compound leaves. This is the first report on the artificial generation of compound leaves from simple leaves in cotton.
\end{abstract}

Keywords Leaf development $\cdot$ LMII-like $\cdot$ KNOXI $\cdot$ Compound leaves

$\begin{array}{ll}\text { Abbreviations } \\ \text { AS1 } & \text { ASYMMETRIC LEAVES1 } \\ \text { AS2 } & \text { ASYMMETRIC LEAVES2 } \\ \text { KNOX1 } & \text { ClasS I KNOTTEDI-like homeobox } \\ \text { CUC } & \text { CUP-SHAPED COTYLEDON } \\ \text { BP } & \text { KNATI/BREVIPEDICELLUS }\end{array}$

Lijing Chang and Gaofu Mei have contributed equally to this work.

Electronic supplementary material The online version of this article (https://doi.org/10.1007/s11103-019-00829-7) contains supplementary material, which is available to authorized users.

Tianzhen Zhang

cotton@njau.edu.cn

1 State Key Laboratory of Crop Genetics and Germplasm Enhancement, College of Agriculture, Nanjing Agricultural University, Nanjing 210095, China

2 Crop Science Institute, Agronomy Department, College of Agriculture and Biotechnology, Zhejiang University, Zhejiang 310029, China

$\begin{array}{ll}\text { KNAT } & \text { Knotted in A. thaliana } \\ \text { Ler } & \text { Landsberg erecta } \\ \text { LMII } & \text { LATE MERISTEM IDENTITY1 } \\ \text { ML } & \text { Maximum likelihood } \\ \text { RCO } & \text { REDUCED COMPLEXITY } \\ \text { SAM } & \text { Shoot apical meristem } \\ \text { STM } & \text { SHOOT MERISTEMLESS } \\ \text { TL } & \text { Tendril-less } \\ \text { VIGS } & \text { Virus induced gene silencing } \\ \text { WT } & \text { Wild-type }\end{array}$

\section{Introduction}

Plant leaves are initiated from the peripheral region of the shoot apical meristem (SAM), and display great variations in shape and size. They are traditionally classified into two major morphogenetic classes: undivided simple leaves, and compound leaves. Simple leaves have a single lamina that can sometimes be elaborated with less-pronounced incisions such as serrations or lobes on the laminal margin, 
for example, unlobed leaves in Arabidopsis and broad or lobed leaves in cotton (Fig. 1a-c). Compound leaves or dissected leaves have numerous individual leaflets on a rachis that arises at a node, for example, ternate compound leaves of soybean and pinnately compound leaves of tomato (Fig. 1d, e). The same factors are involved in the formation of serrations and leaflets, and include auxin activity maxima and CUP-SHAPED COTYLEDON (CUC) genes (Blein and Laufs 2008; Kougioumoutzi 2008; Bilsborough et al. 2011; Kasprzewska et al. 2015). Many compound leaf mutants in Cardamine hirsuta and Medicago truncatula have defective separation between the adjacent leaflets leading to the conversion of dissected leaves into lobed simple leaves (Peng et al. 2011; Vlad et al. 2014). Lobed simple leaves are essentially an intermediate shape between serrations and dissected leaves. However, whether gene mutations can cause lobed leaves to become compound leaves requires further investigation.

Cotton plants have a variety of leaf shapes, including broad leaves, as in TM-1, and lobed leaves, as in Okra (Fig. 1b, c). We have found that an $H D-Z I P$ I transcription factor (GhOKRA) controls the formation of deep lobes in cotton (Gossypium) (Chang et al. 2016), which has been independently confirmed by two other groups (Andres et al. 2014, 2016; Zhu et al. 2016). The mutant of GhOKRA in TM-1 results in the production of broad leaves. Compound leaves are non-existent in cotton. The $\varepsilon$ clade of $H D$-ZIP I transcription factors were conserved to regulate leaf shape in many plants (Hofer et al. 2009; Vlad et al. 2014). The GhOKRA homolog, LATE MERISTEM IDENTITY1 (LMII), was first reported in Arabidopsis thaliana as a floral regulator and was found to influence leaf morphogenesis (Saddic et al. 2006). Other GhOKRA homologs, such as REDUCED COMPLEXITY (RCO) in C. hirsuta and Tendril-less (TL) in pea, which are also LMIIlike genes, are emerging as regulators of lateral organ genesis in compound leaves by affecting lateral organ formation (Hofer et al. 2009; Vlad et al. 2014). The function of these LMI1-like genes appears to be similar, such as blade growth-repression in compound leaves and lobed simple leaves (Vlad et al. 2014; Andres et al. 2016).

Class I KNOTTED1-like homeobox (KNOX1) transcription factors are involved in the maintenance of indeterminate cell fate in the SAMs and developing primordia of complex leaves (Bharathan et al. 2002; Hake et al. 2004; Uchida et al. 2010). The KNOX1 genes are expressed in SAM to maintain the indeterminate nature of meristem cells, and are down-regulated at the position where leaf primordia initiate (Lincoln et al. 1994; Hay and Tsiantis 2010; Sluis and Hake 2015). At the site of leaf initiation, $K N O X 1$ genes are downregulated, and auxin signaling occurs. Auxin regulates $K N O X$ expression (Hay et al. 2006), and KNOX modulates many genes in auxin signaling (Bolduc et al. 2012); thus, they have regulatory interactions. The expression of $K N O X 1$ genes is essential in the developing primordia for leaflet formation in compound leaf plants. However, in simple leaf plants, the $K N O X 1$ genes are absent from leaf primordia (Parnis 1996; Hay and Tsiantis 2006; Kougioumoutzi 2008; Efroni and Lifschitz 2010). Compound leaf development requires organogenic activity during primary morphogenesis and leaflet formation. The KNOX1 genes can be classified into three subclasses; SHOOT MERISTEMLESS (STM)-like, Knotted in A. thaliana (KNAT)2/6-like and KNATI/BREVIPEDICELLUS(BP)-like. In A. thaliana, ASYMMETRIC LEAVES1 (AS1) and AS2 repress the activity of KNAT1 and KNAT2 in leaves (Byrne et al. 2000, 2002). Both as 1 and as 2 mutants have abnormal lobed leaves with ectopic expression of BP and KNAT2 (Byrne et al. 2000, 2002). The MYB transcription factors, which are encoded by AS1, interact with the LATERAL ORGAN BOUNDA$\operatorname{RIES}(L O B)$ domain protein, AS2, and work together as the AS1-AS2 complex. This complex binds $K N O X$ loci, resulting in the recruitment of chromatin remodeling factors, such as HDA6, a histone deacetylase (Luo et al. 2012; Lodha et al. 2013). Both BTB ankyrin genes, BLADE ON PETIOLE1 (BOP1) and BOP2, can activate AS2 directly, but also can repress $K N O X$ independently (Khan et al. 2014). Some simple leaves develop from complex primordia through
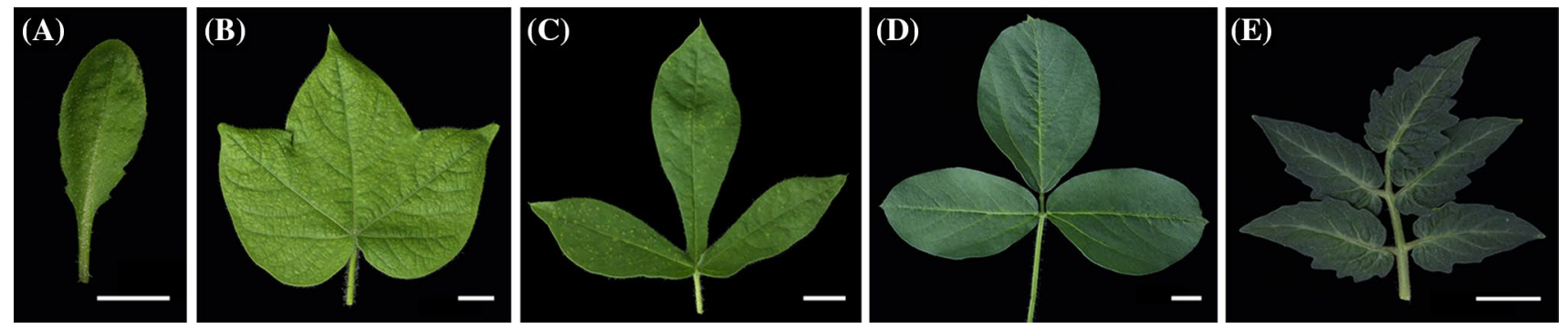

Fig. 1 A variety of leaf shapes in dicotyledons. a A. thaliana, Col-0, b G. hirsutum acc. TM-1, c G. hirsutum acc. Okra cotton, d Glycine max, Williams 82, and e Lycopersicon esculentum, Micro-Tom. Scale bars $=1 \mathrm{~cm}$. Okra cotton was used in VIGS assays for investigating the effect of silencing GhAS1, GhAS2, KNOX1 and GhOKRA genes. TM-1 cotton was used in GhAS1 and GhAS2 silencing VIGS assays. Transgenic A. thaliana (Col-0) constitutively expressed GhOKRA resulted in the production of a lobed leaf 
secondary morphogenesis, as in Lepidium oleraceum, which has KNOX1 expression in leaf primordia to produce marginal outgrowths (Bharathan et al. 2002). In addition, ectopic expression of KNATI in A. thaliana transforms simple leaves into lobed leaves, resulting in an increase in leaf complexity (Chuck et al. 1996). In Cardamine hirsute, which has dissected leaves comprising leaflets, a KNOXI paralogous gene, $\mathrm{Ch} B P$, is concurrently regulated by the microRNA164A (MIR164A)/ChCUP-SHAPED COTYLEDON (ChCUC) module and ChASYMMETRIC LEAVES1 (ChASl). This gene does not occur in A. thaliana, a relative of Cardamine hirsute, which has simple leaves (Rastsomssich et al. 2015). However, it is still unclear whether KNOXI genes regulate lobe development in simple leaves. Organogenic activity and leaf marginal structure development are known to dictate the final leaf shape; however, there is little information on the role of KNOX1 genes in combination with LMII-like genes in the formation of different leaf shapes.

To determine whether the LMII-like gene regulates leaf shape in collaboration with $K N O X 1$ genes, we silenced the KNOX1 genes and the homologous genes of AS1 and AS2 in wild-type (WT) okra leaf cotton, and found that different expression models of the LMII-like gene and KNOXI genes control the formation of broad, lobed and compound leaves. We also generated dissected-like leaves in A. thaliana, which further confirms that formation of these leaf shapes is controlled by LMII-like gene and KNOXI genes. The present research provides new insights into the formation of different leaf marginal structures, including unlobed leaves, lobed leaves, and leaflets.

\section{Materials and methods}

\section{Plant materials}

TM-1 with broad leaves is a standard genetic line of Upland cotton (Kohel et al. 1970). Okra cotton with okra leaves, provided by the Institute of Cotton Research of CAAS named as Super Okra, is a Gossypium hirsutum accession. The A. thaliana mutants used in this study, as 1-101 and as 2-101, were produced with a Landsberg erecta (Ler) background. as I101 and as 2-101 seeds were kindly provided by Lin Xu and Hai Huang (Institute of Plant Physiology \& Ecology, SIBS, CAS) (Yue et al. 2000; Sun et al. 2002; Xu et al. 2002). Transgenic A. thaliana constitutively expressing GhOKRA plants were generated previously in a Col-0 background (Chang et al. 2016). The T1 progeny of transgenic A. thaliana with lobed leaves was crossed with as 1-101 and as2-101 mutant plants. The leaves of the $\mathrm{F}_{1}$ progeny had more lobes than that observed in constitutively expressing GhOKRA A. thaliana plants. There were many different leaf phenotypes in the $\mathrm{F}_{2}$ populations of these two crosses, such as wild-type, as 1-101 or as 2-101, and constitutively expressed GhOKRA A. thaliana leaves, as well as some novel leaf types. All materials were grown in the green houses of Nanjing Agriculture University following normal practices.

\section{Phylogenetic analyses of KNOX genes in cotton}

The genes in Gossypium raimondii are highly homologous with those in G. hirsutum. Since G. hirsutum has two subgenomes, and some homologous have not been annotated in the G. hirsutum acc. TM-1 genome, so we analyzed KNOX genes in diploid $G$. raimondii for next work. G. raimondii genome sequences were downloaded from the Phytozome database (http://www.phytozome.net) (Paterson et al. 2012). Arabidopsis thaliana KNOX protein sequences were downloaded from the Arabidopsis Information Resource website (TAIR) (http://www.arabidopsis.org). All cotton proteins were screened for potential KNOX genes using HMMER software version 3.0 and the Pfam database (Zhang and Wood 2003; Finn et al. 2011, 2016). KNOX protein sequences from $G$. raimondii were aligned with the homologous proteins from A. thaliana. We used the Maximum likelihood (ML) method to construct a phylogenetic tree in MEGA 6.06 (http://www.megasoftware.net). The bootstrap test of phylogeny was performed with 1000 replications (Tamura et al. 2013).

\section{Cloning of GhAS1, GhAS2 and KNOX1 genes}

Homologs of AS1 and AS2 in A. thaliana were identified using HMMER software version 3.0 and the Pfam database to screen all TM-1 proteins (Zhang and Wood 2003; Finn et al. 2011, 2016). We cloned the coding sequences (CDSs) of GhAS1, GhAS2 and some KNOX1 genes from shoot tips of okra cotton using the genome sequence of TM-1 (Zhang et al. 2015). The primers used are listed in Table S1. We used ExTaq DNA Polymerase (TaKaRa, Japan) for PCR. Amplification products were cloned into the pMD19-T vector (TaKaRa, Japan) for sequencing by the Nanjing Jinsite Biotech. Co. Ltd. All of the genes mentioned above were cloned from okra cotton, with separation of the A and the D sub-genomes. Based on the genome sequences of G. raimondii and G. arboreum and the sub-genome sequences in G. hirsutum, we differentiated between the A and the D subgenome homologs (Table S2). Both the A and the D subgenome homologs in G. hirsutum are highly homologous with those in G. raimondii. Only a few SNPs exist between them. A silencing construct developed from the A or the D sub-genome would silence two sub-genome homeologs. We therefore chose only one sub-genome for further analysis due to the high sequence similarity of the $\mathrm{A}$ and the $\mathrm{D}$ sub-genomes. 


\section{Virus-induced gene silencing (VIGS) assay}

We amplified fragments around 300-bp long from the 3' ends of GhAS1, GhAS2 and seven KNOX1 genes (GhKNL2GhKNL8) (Table S2). A ClonExpress II One Step Cloning Kit (C112-01, Vazyme Biotech Co., Ltd) was used to recombine the fragments into EcoRI-XbaI-digested pTRV2 for VIGS assay. All primers used for VIGS vector construction are listed in Table S1. The VIGS assay was carried out according to methods described previously (Liu et al. 2002). Cotton seedlings were grown in a growth chamber $\left(21-25^{\circ} \mathrm{C}\right)$ under long days light cycle $(16 \mathrm{~h}: 8 \mathrm{~h}$, light:dark). The Agrobacterium mixture was infiltrated into the abaxial side of cotyledons of 8-day-old cotton seedings by needleless syringes. More than 15 Okra cotton individuals with okra leaves were infiltrated with pTRV2-GhAS1 or pTRV2GhAS2. Around 15 more TM-1 individuals with broad leaves were infiltrated with pTRV2-GhAS1 or pTRV2-GhAS2. More than 15 Okra cotton individuals with okra leaves were infiltrated with mix of pTRV2-GhKNLs (GhKNL2GhKNL8). We mixed different KNOX1 genes together in VIGS assays to inhibit functional complementation between paralogous genes as follows; GhKNL2 and GhKNL3 with GhKNL4, GhKNL5 with GhKNL6, and GhKNL7 with GhKNL8. These KNOX1 gene combinations were chosen based on relationships represented by phylogenetic tree (Fig. 2). KNOX1 genes can be classified into three subclasses. We chose these genes belong to the same subclass as a combination. pTRV2 was used as a negative control. Three independent tests were carried out for all VIGS assays.

\section{Quantitative real time PCR (qPCR) analysis}

To detect the expression of silenced genes (GhOKRA and $G h K N L 2 / 3 / 4$ ), the shoot tips (approximately $6 \mathrm{~mm}$ ) of Okra cotton individuals infiltrated with either pTRV2-GhAS1 or pTRV2-GhAS2 at the same growth stage were harvested by wiping off the same number of leaves when the leaf shape had changed. We used the Biospin Plant Total RNA Extraction Kit (BioFlux, cat: BSC65S1) to extract total RNA from the shoot tips. First-strand cDNA was generated according to the manufacturer's instructions using TransScript ${ }^{\circledR}$ One-Step gDNA Removal and cDNA Synthesis SuperMix (TransGen Biotec Co., Ltd., cat: AT311) kits. The cotton Histone 3 gene (His3, GenBank accession number: AF024716) was used as a reference gene. The qPCR primer sequences are listed in Table S1. They are universal for both the $\mathrm{A}$ and the $\mathrm{D}$ sub-genome homologs. So, we can detect all the two sub-genome homeologs. qPCR products were quantified according to the manufacturer's instructions using the ABI 7500 Real Time System (Applied Biosystems, USA) and the light cycler fast start DNA Master SYBR Green I kit (Roche, Basel, Switzerland).

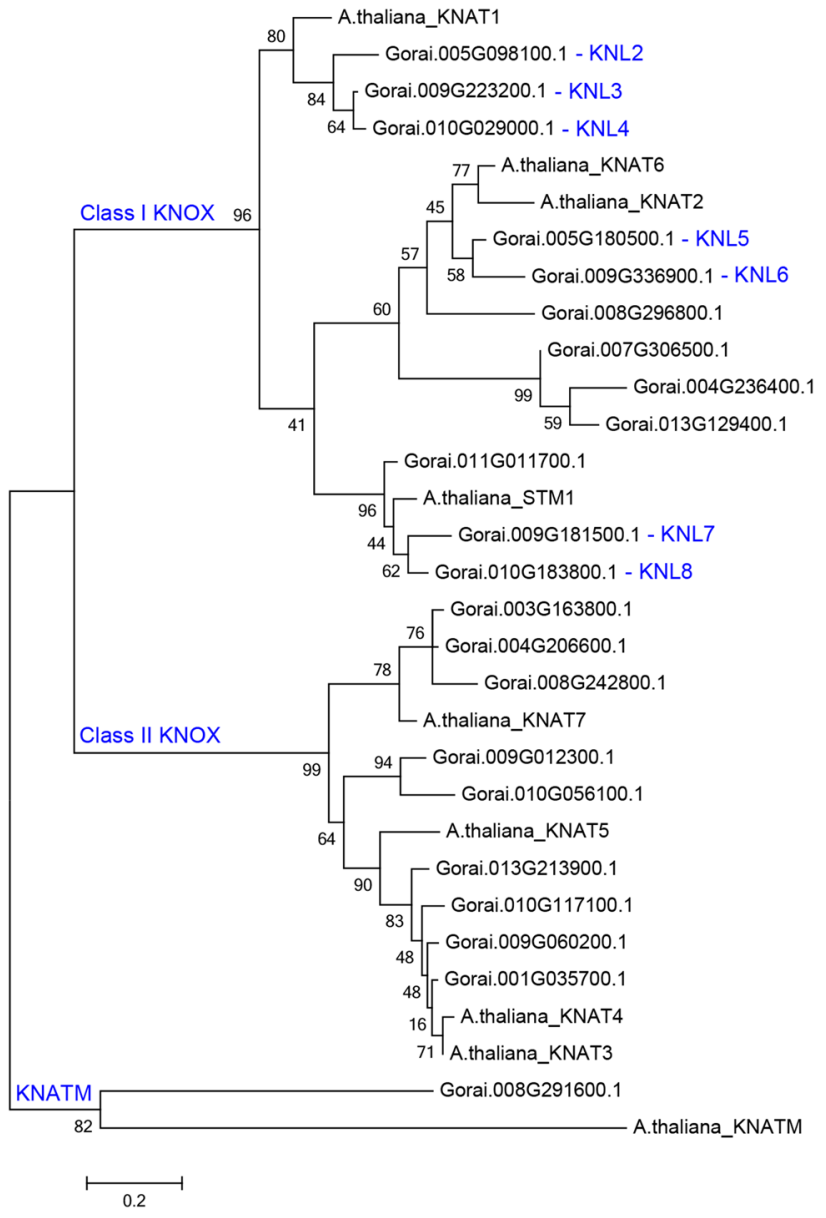

Fig. 2 Phylogenetic tree of KNOX genes of Arabidopsis thaliana and Gossypium raimondii. A maximum-likelihood tree was generated by aligning KNOX protein sequences of $G$. raimondii and A. thaliana. $G$. raimondii KNOX proteins were classified into three clades; class I KNOX, class II KNOX and KNATM. Seven KNOXI genes were selected and named GhKNL2-GhKNL8 in this study. Phylogenetic analysis was carried out using the ML method with 1000 resampling replicates

\section{Results}

\section{Plants silenced in either GhAS1 or GhAS2 produce compound leaves}

To explore the relationship between an HD-ZIP I transcription factor (GhOKRA) and other genes in modulating leaf development, we cloned the corresponding cotton homologs of ASYMMETRIC LEAVES1 (AS1), AS2 and seven $K N O X 1$ genes that regulate leaf development in A. thaliana (Table S2). Based on known KNOX gene domains, 22 KNOX genes were identified in the G. raimondii genome (Fig. 2). Of them, seven cotton class I $K N O X$ genes, named GhKNL2 (KNOTTEDI-LIKE) to GhKNL8, that have close relationships with the corresponding 
homologs in A. thaliana were cloned from the wild-type (WT) okra leaf cotton (Fig. 2) (Gong et al. 2014).

There was only one corresponding homolog of either $A$. thaliana AS1 or AS2 in each sub-genome in cotton. To our knowledge, there are no reports on how these two genes regulate leaf development in cotton. In the GhAS1 and GhAS2 virus induced gene silencing (VIGS) assays, the leaves of all okra leaf plants infiltrated with either pTRV2-GhAS1 or pTRV2-GhAS2 became split from the petiolar sinus and dissected 3 weeks after infiltration (Fig. 3a). Leaf blades at the base of the leaf vein disappeared. Three individual petiolulate leaflets arose at a new distinct node. Each leaflet had a long petiole (Fig. 3a, e). The deeply lobed leaves in the okra leaf cotton were replaced by ternate compound leaves; a phenomenon that has not been observed before in cotton. qPCR analysis showed that the expression of GhAS1 and GhAS2 was lower in pTRV2-GhAS1 and pTRV2-GhAS2silenced lines compared to controls (Fig. 3b). It is clear that the lobed simple leaves of okra cotton were transformed into compound leaves when either GhAS1 or GhAS2 expression was suppressed. These results demonstrate that GhAS1 and GhAS2 regulate the depth of leaf lobes in cotton. GhAS1 may be involved in leaf adaxial/abaxial polarity in cotton, since individual plants infiltrated with pTRV2-GhAS1 exhibited rumpled and curled leaves (Fig. 3a).

We used VIGS to explore how GhOKRA modulates leaf development. One month after infiltration with pTRV2 fused with KNOX1 genes (GhKNL2-GhKNL8), the WT okra leaf cotton retained its okra leaf shape (Fig. 4i-1); however, KNOX1 gene-silenced plants grew slowly (Fig. 4a-d), leaves in their shoot apex became compact and the distance between nodes was shorter than in controls (Fig. 4e-h, m). From these experiments, we could not reveal the roles of KNOX1 in lobe development in cotton.

\section{GhOKRA is necessary for compound leaf formation}

GhOKRA controlled okra leaf development in the WT cotton. Ectopic expression of the wild cotton GhOKRA gene under the control of the 35S promoter (Pro35S::GhOKRA) led to a lobed leaf type rather than compound leaves in $A$. thaliana (Chang et al. 2016). To explore whether GhASI and $G h A S 2$ regulate the depth of leaf lobes in collaboration with the GhOKRA gene, we silenced either GhASl or GhAS2 in both WT okra leaf cotton (Okra cotton) and mutant broad leaf cotton (TM-1). All GhAS1 or GhAS2-silenced WT okra cotton plants had larger lobe depths than controls, suggesting that the functions of GhAS1 and GhAS2 are the same in leaf lobe development. $A S 1$ and $A S 2$ promote leaf adaxial-abaxial polarity specification and repress $K N O X$ gene expression by forming AS1-AS2 protein complexes in A. thaliana ( $\mathrm{Fu}$ et al. 2007; Guo et al. 2008). Therefore, we suppose that the AS1-AS2 complex might influence leaf lobe development as an upstream regulator of GhOKRA. All GhAS1 or GhAS2silencing in broad leaf TM-1 plants caused no change in the leaf lobes, although the second and third leaves of GhASIsilenced plants were seriously curled (Fig. S1). The broad leaf TM-1 had a mutated non-functional Ghokra genotype which led to a different result compared with Okra cotton after silencing of GhAS1 or GhAS2. These results suggest that GhOKRA is necessary for compound leaf formation.

\section{GhKNL2/3/4 expression was elevated in either GhAS1 or GhAS2-silenced plants}

To demonstrate whether the AS1-AS2 complex negatively regulates GhOKRA expression, the transcript levels of GhOKRA in either GhAS1 or GhAS2-silenced individuals were detected. There was no difference in the expression levels of GhOKRA in either GhAS1 or GhAS2-silenced plants compared to the negative control (Fig. 3d), confirming that the AS1-AS2 complex does not regulate GhOKRA. The AS1-AS2 protein complex can repress Knotted in A. thaliana 1 (KNAT1) and KNAT2 activity in leaves (Guo et al. 2008). When KNAT1 was overexpressed in A. thaliana, the leaves showed ectopic stipules (Chuck et al. 1996). The transcript levels of GhKNL2/3/4, which are homologs of KNAT1, were thus analyzed in the shoot tips of either GhASl or GhAS2-silenced plants. Compared with the negative control, the expression levels of all three homologs were elevated in the either GhAS1 or GhAS2-silencing plants (Fig. 3c), with a greater increase observed in the GhAS2-silenced plants. These results suggest that GhAS1 and GhAS2 repress the expression of KNOXI in leaf primordia of cotton and this repression might be responsible for the change from lobed simple leaves to compound leaves in the GhAS1 or GhAS2silenced plants.

\section{Co-expression of GhLMI1-like and KNOX1 genes produces compound-like leaves in A. thaliana}

In A. thaliana, the GhOKRA homolog was secondarily lost through duplication, leading to the evolution of a simple leaf (Vlad et al. 2014). Previously, we generated constitutively expressing GhOKRA A. thaliana plants with a lobed leaf (Chang et al. 2016). To determine whether coordination of the LATE MERISTEM IDENTITY 1 (LMII)-like and $K N O X 1$ genes influences the formation of different leaf shapes, we crossed heterozygous constitutively expressing GhOKRA A. thaliana plants with homozygous as 1-101 and as 2-101 mutant plants in a Landsberg erecta (Ler) background (Yue et al. 2000; Sun et al. 2002; Xu et al. 2002). As compared to the WT type A. thaliana (Fig. 5a), the leaves of as 1-101 plants were heart-shaped and the leaf edges were slightly curled down with no lobes (Fig. 5b), while the mutant as2-101 rosette leaves were broad (Fig. 5c). $\mathrm{F}_{1}$ 
(A)

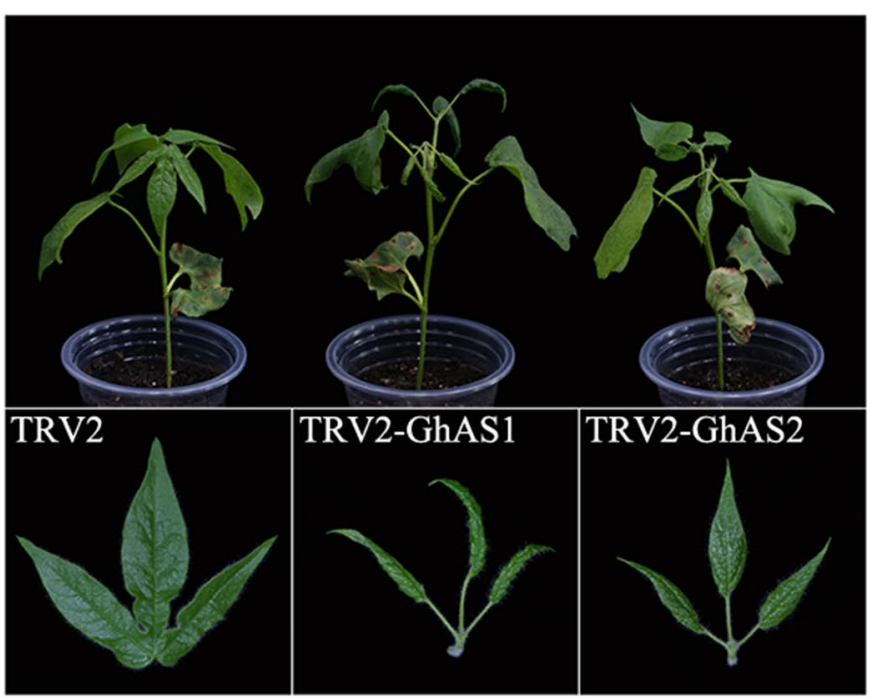

(B)

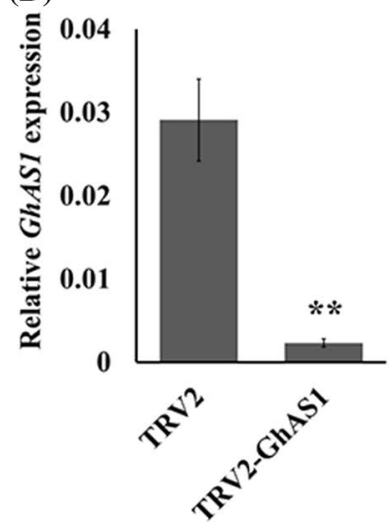

(D)

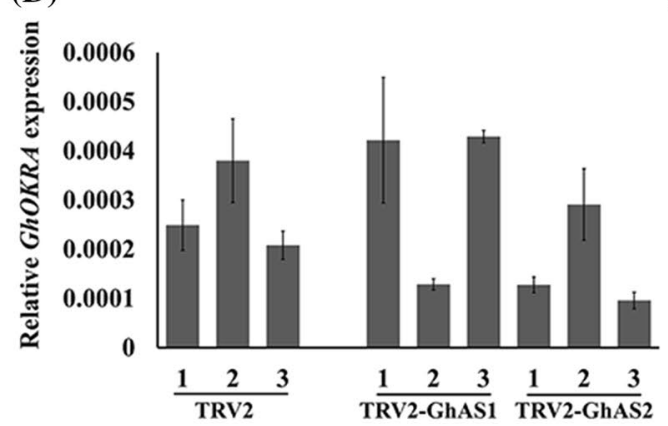

Fig. 3 Leaf phenotypes of VIGS GhAS1 or GhAS2- silenced okra cotton plants. a Plant phenotypes from left to right: pTRV2, pTRV2GhAS1 and pTRV2-GhAS2. Leaf shape of negative control plants (left), okra cotton plants silenced by pTRV2-GhAS1 (middle), and okra cotton plants silenced by pTRV2-GhAS2 (right). The leaf lobes deepened and the blade at the base of the leaf vein disappeared in either $G h A S 1$ or $G h A S 2$-silenced plants. b GhAS1 transcript levels in $G h A S 1$-silenced and negative control plants. GhAS2 transcript levels in $G h A S 2$-silenced and negative control plants. c GhKNL2, GhKNL3, and GhKNL4 transcript levels in GhAS1 or GhAS2-silenced and negative control plants. The expression of GhKNL2, GhKNL3, and viduals
(C)
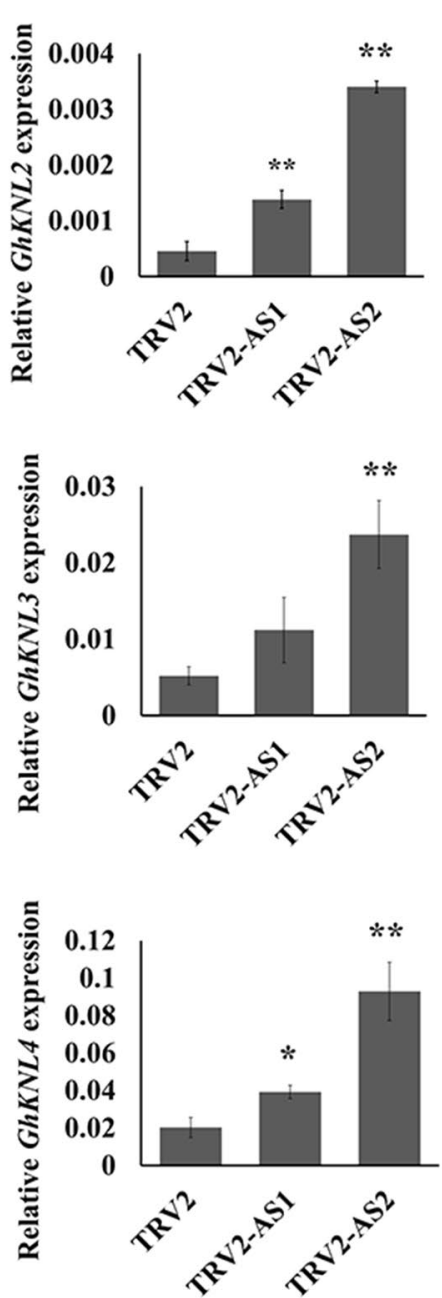

(E)

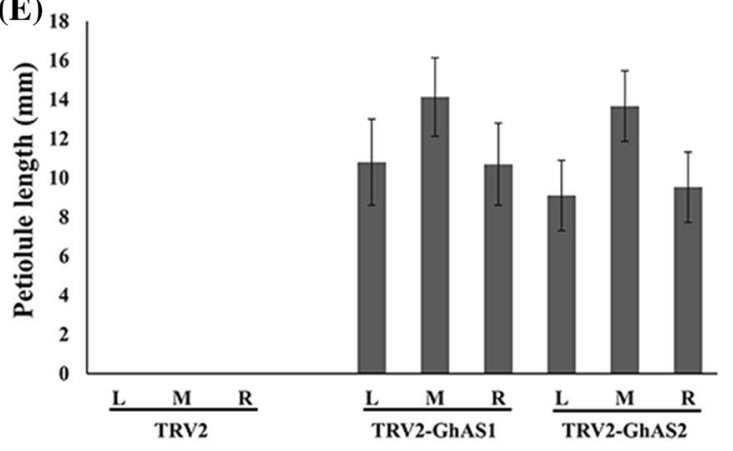

GhKNL4 was higher in silenced cotton than in controls. d GhOKRA transcript levels in three individuals silenced either GhAS1 or GhAS2 and negative control. There is no significant difference in comparision with the negative control. Data represent the means \pm SDs of three replicated experiments. Asterisks indicate a significant difference at $P<0.05$; Double asterisks indicate a significant difference at $P<0.01$. e We measured the length of the formed left $(\mathrm{L})$, middle (M) and right (R) petiolules in the fourth leaf of the VIGS plants in one of our VIGS assays by 37 days post infiltration (dpi). There is no petiolules in the control. Data represent the means \pm SDs of 15 indi- 

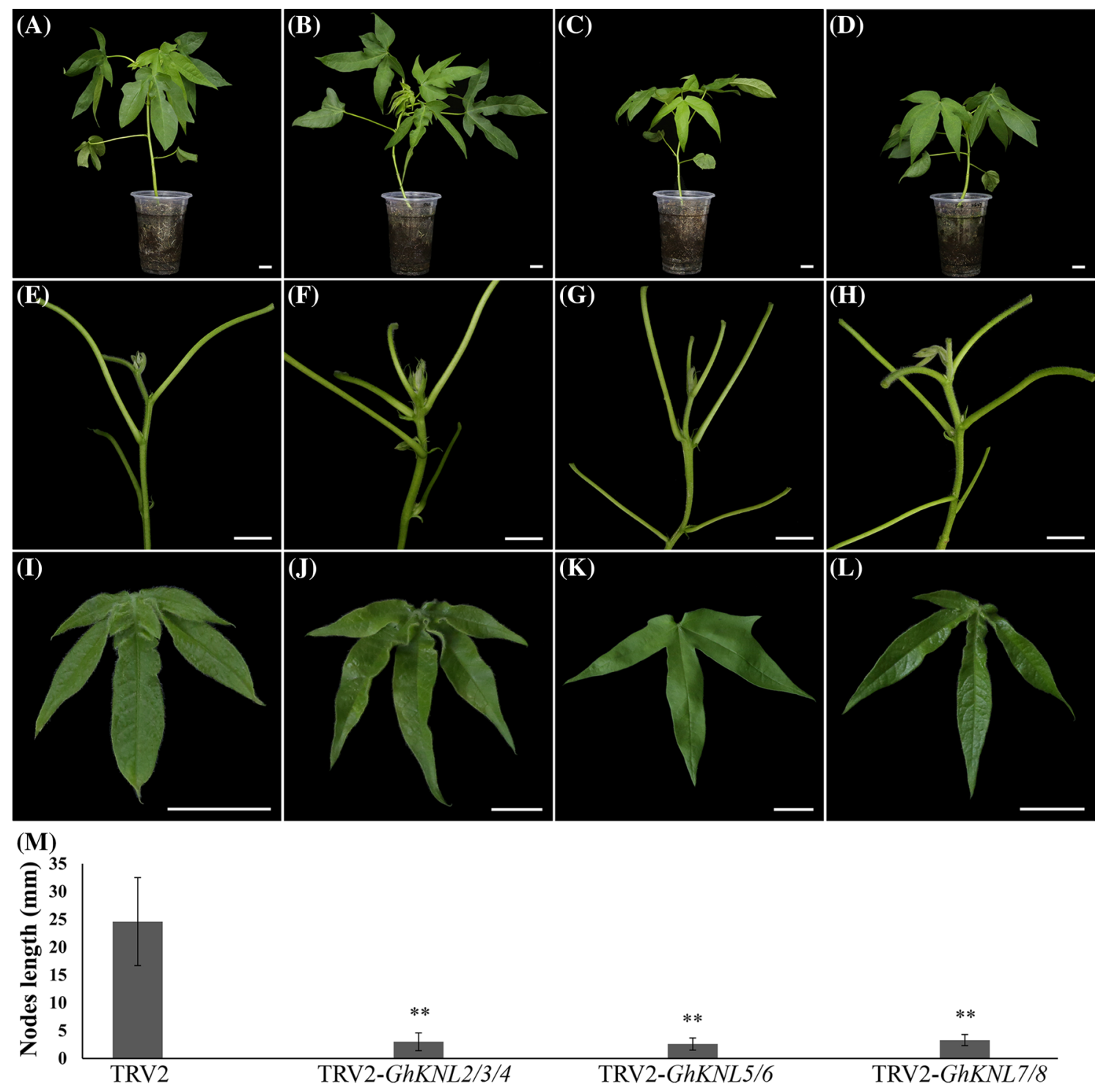

Fig. 4 The phenotype of okra cotton silenced by several paralogous genes from the KNOX1 family. Plant phenotypes from left to right: pTRV2, pTRV2-GhKNL2/3/4, pTRV2-GhKNL5/6, and pTRV2GhKNL7/8. a-d Whole plant scaning; e-h feature of the distance between the nodes; $\mathbf{i}-\mathbf{l}$ Feature of the first leaf. $\mathbf{m}$ We measured the length of the nodes between fifth and sixth nodes of the VIGS plants

plants with a greater number of lobes than constitutively expressing GhOKRA A. thaliana plants were self-pollinated to generate $\mathrm{F}_{2}$ populations. The $\mathrm{F}_{2}$ segregated out several novel leaf types (Table S3) that were different from the WT, as 1-101 and as2-101 mutant parents, and constitutively expressing GhOKRA A. thaliana plants. In Fig. 5, we show some typical individuals with novel leaf types from the two $F_{2}$ populations. Their genotypes were identified by cloning and sequencing (Figs. S2, S3). The rosette leaves of three $\mathrm{F}_{2}$ individuals genotyped as GhOKRA/asl-101 were divided into two or three parts along the proximal-distal in one of our VIGS assays by $61 \mathrm{dpi}$. Data represent the means \pm SDs of 15 individuals. Double asterisks indicate a significant difference at $P<0.01$. Plants with pTRV2-GhKNLs grew slowly and their leaves became compact in shoot apex of cotton, the distance between the nodes were decreased in comparision with the negative control, while the leaf shape showed no change. Scale bars $=2 \mathrm{~cm}$

axis (Fig. 5e-g). Their leaf lobes extended to leaf petioles. Some of their cauline leaves became compound-like leaves (Fig. 5i-k) and leaf-like structures were also observed at the petioles of cauline leaves (Fig. 5j). One individual from the cross between the as2-101 and constitutively expressing GhOKRA A. thaliana plants showed extremely deeply lobed leaves (Fig. 5h), and their secondary lobes appeared on lobed leaves, thus increasing the leaf complexity (Fig. 51).

To further confirm whether these novel leaves were formed from one divided leaf or the fusion of several leaves, we analyzed the leaf vein and found a difference between 


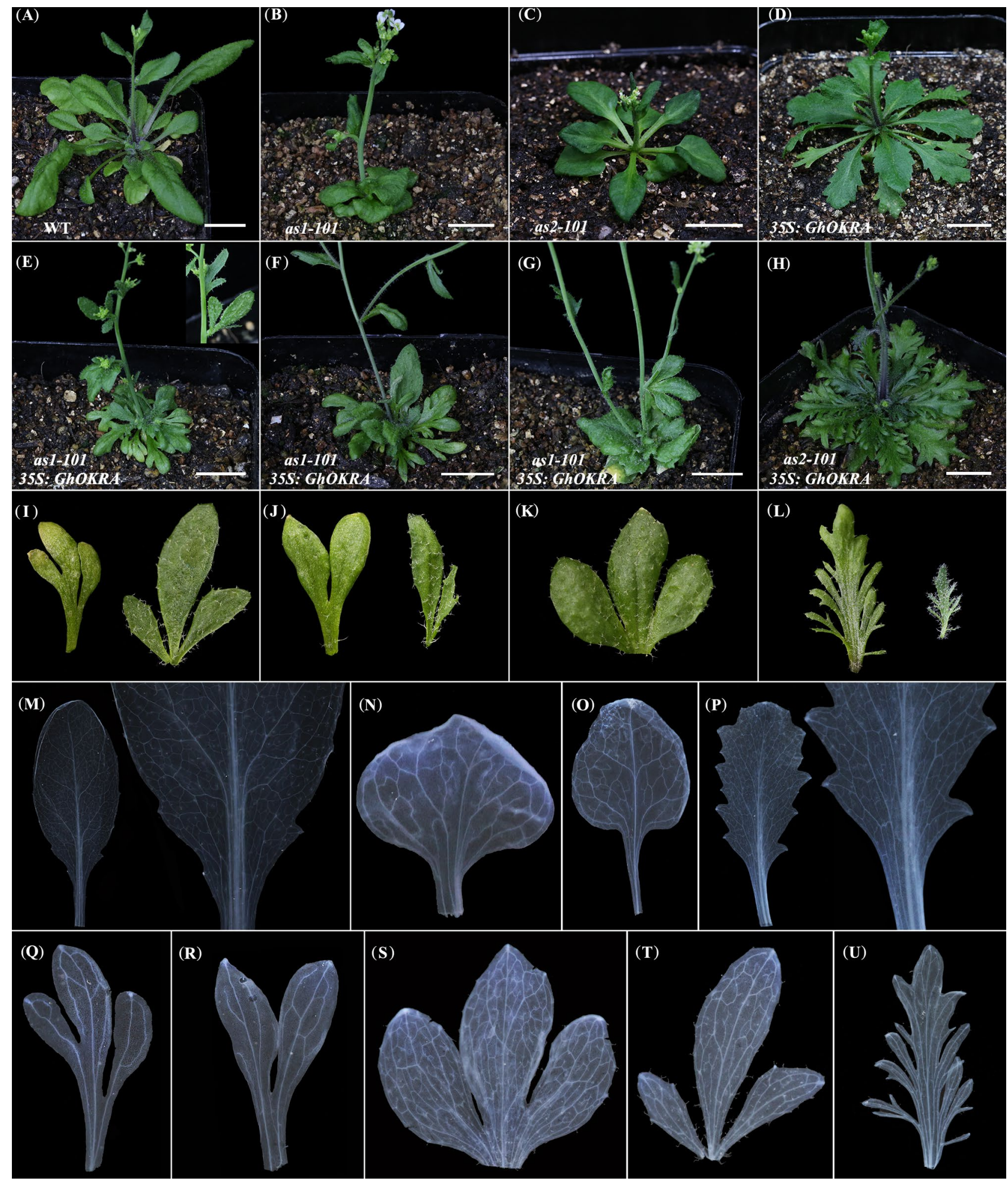

rosette leaves and cauline leaves in the $\mathrm{F}_{2}$ generated by crossing as 1-101 and constitutively expressing GhOKRA A. thaliana. The aberrant rosette leaves in $\mathrm{F}_{2}$ plants had a reduced vascular structure. Lobes were divided between main veins, which indicates that the leaf was formed from a single complete leaf (Fig. 5q, r). The cauline leaves from $\mathrm{F}_{2}$ plants had a complex vascular structure. Each leaflet vein was similar to those of wide-type leaves, which suggests that these cauline leaves became compound-like leaves through the fusion of simple leaves (Fig. $5 \mathrm{~s}$, t). 
४Fig. 5 Phenotypes and leaf vein patterns of parents and their $\mathrm{F}_{2}$ individuals. a-d Morphological observations of wild-type Col-0 (a), mutant as 1-101 (b), mutant as2-101 (c) and constitutively expressing GhOKRA A. thaliana (d) plants. e- $\mathbf{g}$ The $\mathrm{F}_{2}$ individuals of as 1 101 and constitutively expressing GhOKRA A. thaliana plants. h The $\mathrm{F}_{2}$ individuals of as2-101 and constitutively expressing GhOKRA A. thaliana plants. $\mathbf{i}-\mathbf{k}$ The rosette leaf (left) and cauline leaf (right) of $\mathrm{F}_{2}$ individuals of as1-101 and constitutively expressing GhOKRA A. thaliana plants. 1 The rosette leaf (left) and cauline leaf (right) of $\mathrm{F}_{2}$ individuals of as2-101 and constitutively expressing GhOKRA A. thaliana plants. Rosette leaves were divided from leaf petioles, and cauline leaves became compound leaves in the $\mathrm{F}_{2}$ individuals of as 1101 and constitutively expressing GhOKRA A. thaliana plants. The $\mathrm{F}_{2}$ individual of as2-101 and constitutively expressing GhOKRA A. thaliana plants showed extremely deeply lobed leaves with secondary lobes. $\mathbf{m}-\mathbf{p}$ Leaf vascular structure of wild-type Col-0 (m), mutant as1-101 (n), mutant as2-101 (o) and constitutively expressing GhOKRA A. thaliana plants (p). q-t Leaf vascular structure of $\mathrm{F}_{2}$ individuals of as1-101 and constitutively expressing GhOKRA A. thaliana plants. Two different leaf vein patterns of $\mathrm{F}_{2}$ individuals indicated different methods of leaflet-like rosette and cauline leaf formation. u Leaf vascular structure of $\mathrm{F}_{2}$ individuals of as2-101 and constitutively expressing GhOKRA A. thaliana plants. Scale bars $=1 \mathrm{~cm}$

The vascular structure of rosette leaves of $\mathrm{F}_{2}$ plants from the cross between as $2-101$ and constitutively expressing GhOKRA A. thaliana was more complex. Secondary lobes increased the complexity of the vascular structure (Fig. 5u).

In the A. thaliana $\mathrm{F}_{2}$ populations generated in this study, the phenotypic segregation did not follow a Mendelian pattern. The constitutively expressing GhOKRA A. thaliana plants showed a deeply lobed leaf in the T1 progeny (Chang et al. 2016), but most had disappeared in the $\mathrm{T}_{2}$ progeny. It is due to the limitations of the heterologous overexpression system which we used. Therefore, the number of progeny with dissected leaves was lower than expected (Table S3).

\section{Discussion}

\section{Temporal regulation of GhOKRA and KNOX1 genes during compound leaf development}

We previously reported that GhOKRA-silenced plants display palmate leaves changed from deeply lobed okra leaves (Chang et al. 2016). Based on the present study, it seems like that elevated expression of KNAT1 homologous genes in lobed leaf primordia results in the formation of dissected leaves. This is the first report of the generation of compound leaves in cotton (Fig. 3a). As we know, transient gene silencing is the limitation of the VIGS method. While the VIGS method seems like have advantages over transgenic plant methods in research into gene temporal regulation for gene function identification. We can explore the temporal expression differences between two genes by observing their phenotypic variation via VIGS. It has been reported that leaf marginal structures, such as leaflets, lobes and serrations, are formed during the phase of primary morphogenesis when leaves are primordia (Bar and Ori 2014). However, in the present study, we observed the phenotypes of leaf marginal structures after the leaves were fully expanded. In our VIGS assay, Okra cotton seedlings were infected with pTRV2-GhAS1, pTRV2-GhAS2 and pTRV2-GhOKRA at the same time. We found that the phenotypes of either GhAS1 or GhAS2 gene-silenced plants were changed from the lobed to compound leaf at the fifth fully expanded leaf (L5). However, in the GhOKRA-silenced plants, the phenotype variations from the lobed to broad leaf were firstly observed at the seventh fully expanded leaf (L7) rather than at L5. These results suggest that silencing of GhAS1 or GhAS2 was effective in the primordium of L5 and those of younger leaves including L6 and L7, whereas silencing of GhOKRA affected leaf morphology only when silencing took place from a very early developmental stage (e.g., primordium of L7). The primordium of L7 is a younger initiating leaf than the primordium of L5. Therefore, it is likely that GhOKRA is involved in leaf primordia development at a very early stage, before $K N O X 1$ genes start to express in the GhAS1 or GhAS2-silensed plants. We speculate that the expression of the LMII-like gene allows lobe formation or limited lamina growth between leaflets first, and then $K N O X 1$ expresses to maintain the indeterminate nature of meristem cells in the developing primordia for leaflet formation (Fig. 6).

\section{LMI1-like and KNOX1 genes may regulate the development of different leaf shapes}

The function of LMII-like genes has been widely reported in different plants (Hofer et al. 2009; Vlad et al. 2014; Ni et al. 2017). Constitutively expressing homologous genes in A. thaliana produced lobed leaves (Chang et al. 2016; $\mathrm{Ni}$ et al. 2017). In the compound leaf plant, Cardamine hirsuta, the homologous gene, $R C O$, repressed lamina growth at the leaf margin (Vlad et al. 2014). However, the molecular context of the LMII-like gene is not clear. KNOX1 transcription factors maintain an indeterminate cell fate in SAMs and complex leaf primordia in most plants (Lincoln et al. 1994; Hay and Tsiantis 2010). Based on the present results, a hypothetical model was proposed to illustrate how the LMIIlike and KNOX1 genes regulate the formation of different leaf shapes, including unlobed leaves, lobed leaves and compound leaves (Fig. 6). If primordia only expressed the LMI1-like gene without $K N O X 1$, the leaf shape was lobed (Andres et al. 2016), as in the WT okra cotton (Fig. 1c), and as shown by constitutively expressing LMII-like genes in A. thaliana (Fig. 5d). Removing the activity of the LMII-like gene from lobed primordia led to an unlobed simple leaf type (Fig. 6), as seen in A. thaliana and TM-1 (Vlad et al. 2014; Chang et al. 2016). The GhAS1 or GhAS2-silenced 


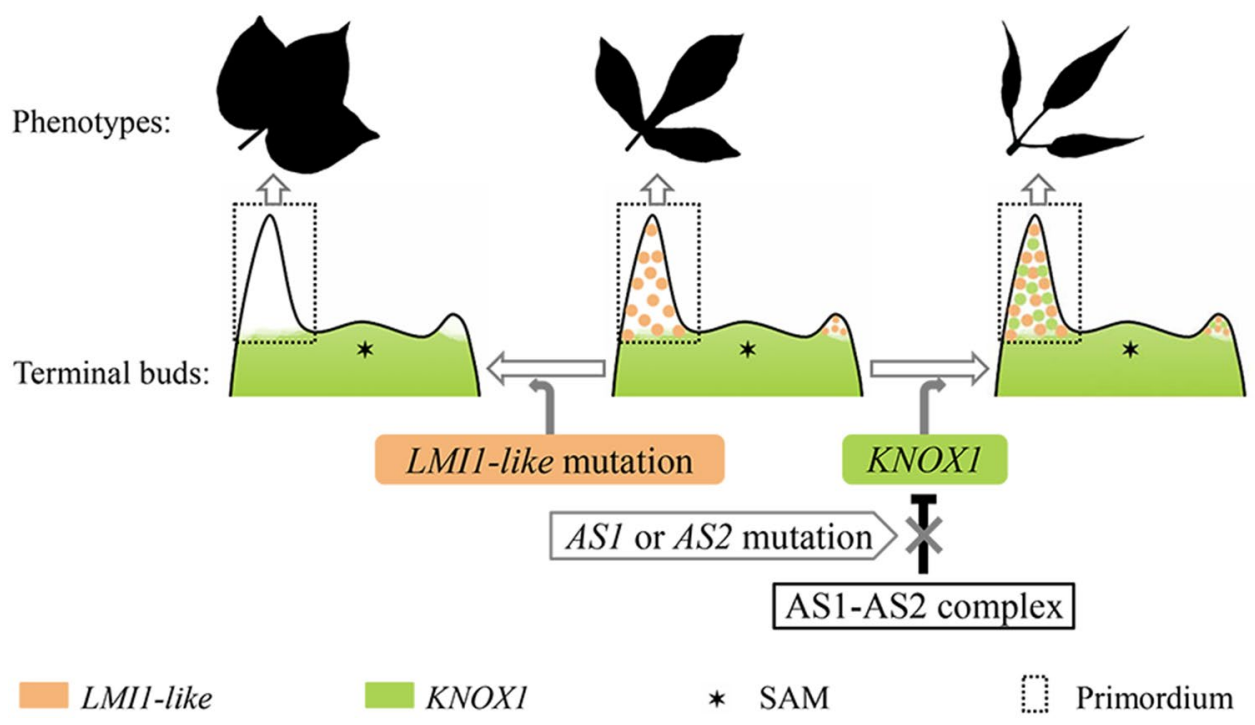

Fig. 6 Hypothesis of regulatory model for the LMI1-like and KNOX1 genes. Different expression patterns of the LMII-like and KNOXI genes regulate the formation of different leaf marginal structures. The primordia of lobed leaf is only developed when the LMI1-like gene express (Andres et al. 2016), but not KNOX1. The inactivity of the LMI1-like gene from lobed primordia will lead to the development of an unlobed simple leaf type (Chang et al. 2016). The AS1-AS2

plants with lobed leaves produce compound leaves (Fig. 3a). As the GhAS1 and GhAS2 repress the expression of KNOX1 (Fig. 3c). So, the KNOX1 expression in lobed primordia might result in compound leaves (Fig. 6). When primordia expressed KNOXI without the LMII-like gene, the leaf shape was either unlobed or lobed, possibly depending on the expression levels of KNOX1 genes. Neither GhAS1 nor GhAS2-silenced TM-1 plants had altered leaf shapes (Fig. $\mathrm{S} 1$ ), while ectopic expression of KNAT1 under the control of the $35 \mathrm{~S}$ promoter in A. thaliana produced lobed leaves (Chuck et al. 1996). Therefore, co-expression of LMI1-like and $K N O X 1$ genes in primordia could result in compound leaves: we generated leaflet-like A. thaliana leaves by crossing as 1-101 and as 2-101 mutants with constitutively expressing GhOKRA plants (Fig. 5). The deeply lobed leaves of $C$. hirsuta rco mutant caused by the fusion of leaflets also supports this hypothetical model (Vlad et al. 2014).

\section{Intricate and complicated factors regulate leaf development}

Many factors repress the activity of $K N O X 1$ in leaf primordia, such as polar auxin transport and TEOSINTEBRANCHE DI/CYCLOIDEA/PROLIFERATING CELL FACTOR (TCP), as well as the AS1-AS2 protein complex (Scanlon 2003; Guo et al. 2008; Li et al. 2012). POLYCOMB REPRESSIVE COMPLEX (PRC) 2 interacts with the AS1-AS2 protein complex to stably silence KNAT1 and KNAT2 in protein complex repress the activity of $K N O X 1$ in leaf primordia, but their mutations can remove this repression. This repression might be responsible for the change from lobed simple leaves to compound leaves in the GhAS1 or GhAS2-silenced plants. Orange mark LMI1like gene; green mark KNOX1 genes; asterisks mark the SAM; dashed panes mark primordia

leaf primordia (Lodha et al. 2013). In addition, in pea, the FLORICAULA/LEAFY ortholog, UNIFOLIATA, rather than $K N O X 1$, was found to control the development of compound leaves (Hofer et al. 1997). It has also been reported that CUC genes define boundaries between leaflets in compound leaf plants (Blein and Laufs 2008; Berger et al. 2009). Leaf development is changeable and complicated and is regulated by many factors. Ectopic -expressing GhOKRA A. thaliana plants develop many lobes in leaves in the same direction along the medial-lateral axis (Chang et al. 2016). The $A$. thaliana $\mathrm{F}_{2}$ progeny showed lobes in two directions; along the medial-lateral and proximal-distal axes (Fig. 5i-1). KNOX1 might affect the position or pattern of GhOKRA expression. The dissected leaves of GhAS1 and GhAS2silenced okra cotton plants indicate that compound leaves are formed from deeply lobed simple leaves (Fig. 3a). There were two types of leaf vein distribution in A. thaliana $\mathrm{F}_{2}$ progeny (Fig. 5q-u), and leaf vein development also affected leaflet formation (Runions et al. 2017). Whether compound leaves are formed from collections of simple leaves deserves further research (Champagne and Sinha 2004). In this study, we propose that the LMII-like and KNOXI genes regulate the formation of several margin structures (Fig. 6).

Acknowledgements This work was financially supported in part by Supported by the earmarked fund for China Agriculture Research System, and the Distinguished Discipline Support Program of Zhejiang University. We thank Prof. Lin Xu and Prof. Hai Huang for A. thaliana mutant seeds of as 1-101 and as 2-101. We thank for Medium-term 
Gene Bank of Cotton in China, Institute of Cotton Research of CAAS providing seeds of Super okra used in the present study.

Author contributions TZZ designed the research; LJC, GFM performed research; TZZ, LJC, GFM analyzed all data and wrote the manuscript. LJC, GFM, JQD constructed VIGS assay. LJC, GFM, YH analyzed qPCR result. GFM constructed A. thaliana crosses. All authors discussed results and commented on the manuscript.

\section{Compliance with ethical standards}

Conflict of interest The authors declare that they have no conflict of interest.

Open Access This article is distributed under the terms of the Creative Commons Attribution 4.0 International License (http://creativeco mmons.org/licenses/by/4.0/), which permits unrestricted use, distribution, and reproduction in any medium, provided you give appropriate credit to the original author(s) and the source, provide a link to the Creative Commons license, and indicate if changes were made.

\section{References}

Andres RJ, Bowman DT, Kaur B, Kuraparthy V (2014) Mapping and genomic targeting of the major leaf shape gene (L) in Upland cotton (Gossypium hirsutum L.). Theor Appl Genet 127:167-177

Andres RJ, Coneva V, Frank MH et al (2016) Modifications to a LATE MERISTEM IDENTITY1 gene are responsible for the major leaf shapes of Upland cotton (Gossypium hirsutum L.). Proc Natl Acad Sci USA 114:E57

Bar M, Ori N (2014) Leaf development and morphogenesis. Development 141:4219-4230

Berger Y, Harpazsaad S, Brand A, Melnik H, Sirding N, Alvarez JP, Zinder M, Samach A, Eshed Y, Ori N (2009) The NAC-domain transcription factor GOBLET specifies leaflet boundaries in compound tomato leaves. Development 136:823

Bharathan G, Goliber TE, Moore C, Kessler S, Pham T, Sinha NR (2002) Homologies in leaf form inferred from KNOX1 gene expression during development. Science 296:1858-1860

Bilsborough GD, Runions A, Barkoulas M, Jenkins HW, Hasson A, Galinha C, Laufs P, Hay A, Prusinkiewicz P, Tsiantis M (2011) Model for the regulation of Arabidopsis thaliana leaf margin development. Proc Natl Acad Sci USA 108:3424-3429

Blein T, Laufs P (2008) A conserved molecular framework for compound leaf development. Science 322:1835-1839

Bolduc N, Yilmaz A, Mejiaguerra MK, Morohashi K, O'Connor D, Grotewold E, Hake S (2012) Unraveling the KNOTTED1 regulatory network in maize meristems. Gene Dev 26:1685

Byrne ME, Barley R, Curtis M, Arroyo JM, Dunham M, Hudson A, Martienssen RA (2000) Asymmetric leaves1 mediates leaf patterning and stem cell function in Arabidopsis. Nature 408:967-971

Byrne ME, Simorowski J, Martienssen RA (2002) ASYMMETRIC LEAVES1 reveals knox gene redundancy in Arabidopsis. Development 129:1957-1965

Champagne C, Sinha N (2004) Compound leaves: equal to the sum of their parts? Development 131:4401-4412

Chang L, Fang L, Zhu Y, Wu H, Zhang Z, Liu C, Li X, Zhang T (2016) Insights into interspecific hybridization events in allotetraploid cotton formation from characterization of a gene regulating leaf shape. Genetics 204:799-806
Chuck G, Lincoln C, Hake S (1996) KNAT1 induces lobed leaves with ectopic meristems when overexpressed in Arabidopsis. Plant Cell $8: 1277$

Efroni I, Lifschitz E (2010) Morphogenesis of simple and compound leaves: a critical review. Plant Cell 22:1019

Finn RD, Clements J, Eddy SR (2011) HMMER web server: interactive sequence similarity searching. Nucleic Acids Res 39:29-37

Finn RD, Coggill P, Eberhardt RY et al (2016) The Pfam protein families database: towards a more sustainable future. Nucleic Acids Res 44:D279-D285

Fu Y, Xu L, Xu B, Yang L, Ling Q, Wang H, Huang H (2007) Genetic interactions between leaf polarity-controlling genes and ASYMMETRIC LEAVES1 and 2 in Arabidopsis leaf patterning. Plant Cell Physiol 48:724-735

Gong SY, Huang GQ, Sun X, Qin LX, Li Y, Zhou L, Li XB (2014) Cotton KNL1, encoding a class II KNOX transcription factor, is involved in regulation of fibre development. J Exp Bot 65:4133

Guo M, Thomas J, Collins G, Timmermans MC (2008) Direct repression of KNOX loci by the ASYMMETRIC LEAVES1 complex of Arabidopsis. Plant Cell 20:48-58

Hake S, Smith HM, Holtan H, Magnani E, Mele G, Ramirez J (2004) The role of knox genes in plant development. Annu Rev Cell Dev B 20:125-151

Hay A, Tsiantis M (2006) The genetic basis for differences in leaf form between Arabidopsis thaliana and its wild relative Cardamine hirsuta. Nat Genet 38:942

Hay A, Tsiantis M (2010) KNOX genes: versatile regulators of plant development and diversity. Development 137:3153-3165

Hay A, Barkoulas M, Tsiantis M (2006) ASYMMETRIC LEAVES1 and auxin activities converge to repress BREVIPEDICELLUS expression and promote leaf development in Arabidopsis. Development 133:3955-3961

Hofer J, Turner L, Hellens R, Ambrose M, Matthews P, Michael A, Ellis N (1997) UNIFOLIATA regulates leaf and flower morphogenesis in pea. Curr Biol 7:581-587

Hofer J, Turner L, Moreau C et al (2009) Tendril-less regulates tendril formation in pea leaves. Plant Cell 21:420

Kasprzewska A, Carter R, Swarup R, Bennett M, Monk N, Hobbs JK, Fleming A (2015) Auxin influx importers modulate serration along the leaf margin. Plant J 83:705-718

Khan M, Xu H, Hepworth SR (2014) BLADE-ON-PETIOLE genes: setting boundaries in development and defense. Plant Sci 215-216:157-171

Kohel RJ, Richmond TR, Lewis CF (1970) Texas Marker-1. Description of a genetic standard for Gossypium hirsutum L. Crop Sci 10:670-671

Kougioumoutzi E (2008) A developmental framework for dissected leaf formation in the Arabidopsis relative Cardamine hirsuta. Nat Genet 40:1136-1141

Li Z, Li B, Shen WH, Huang H, Dong A (2012) TCP transcription factors interact with AS2 in the repression of class-I KNOX genes in Arabidopsis thaliana. Plant J 71:99-107

Lincoln C, Long J, Yamaguchi J, Serikawa K, Hake S (1994) A knotted1-like homeobox gene in Arabidopsis is expressed in the vegetative meristem and dramatically alters leaf morphology when overexpressed in transgenic plants. Plant Cell 6:1859-1876

Liu YL, Schiff M, Dinesh-Kumar SP (2002) Virus-induced gene silencing in tomato. Plant J 31:777-786

Lodha M, Marco CF, Timmermans MCP (2013) The ASYMMETRIC LEAVES complex maintains repression of KNOX homeobox genes via direct recruitment of Polycomb-repressive complex2. Gene Dev 27:596-601

Luo L, Ando S, Sasabe M, Machida C, Kurihara D, Higashiyama T, Machida Y (2012) Arabidopsis ASYMMETRIC LEAVES2 protein required for leaf morphogenesis consistently forms speckles 
during mitosis of tobacco BY-2 cells via signals in its specific sequence. J Plant Res 125:661-668

Ni X, Liu H, Huang J, Zhao J (2017) LMI1-like genes involved in leaf margin development of Brassica napus. Genetica 145:269-274

Parnis A (1996) The making of a compound leaf: genetic manipulation of leaf architecture in tomato. Cell 84:735

Paterson AH, Wendel JF, Gundlach H et al (2012) Repeated polyploidization of Gossypium genomes and the evolution of spinnable cotton fibres. Nature 492:423-427

Peng J, Yu J, Wang H, Guo Y, Li G, Bai G, Chen R (2011) Regulation of compound leaf development in Medicago truncatula by fused compound leaf1, a class M KNOX gene. Plant Cell 23:3929

Rastsomssich MI, Broholm S, Jenkins H, Canales C, Vlad D, Kwantes M, Bilsborough G, Dello IR, Ewing RM, Laufs P (2015) Alternate wiring of a KNOXI genetic network underlies differences in leaf development of $A$. thaliana and C. hirsuta. Gene Dev 29:2391-2404

Runions A, Tsiantis M, Prusinkiewicz P (2017) A common developmental program can produce diverse leaf shapes. New Phytol 216

Saddic LA, Huvermann B, Bezhani S, Su Y, Winter CM, Chang SK, Collum RP, Wagner D (2006) The LEAFY target LMI1 is a meristem identity regulator and acts together with LEAFY to regulate expression of CAULIFLOWER. Development 133:1673-1682

Scanlon MJ (2003) The polar auxin transport inhibitor N-1-Naphthylphthalamic acid disrupts leaf initiation, KNOX protein regulation, and formation of leaf margins in maize. Plant Physiol 133:597-605

Sluis A, Hake S (2015) Organogenesis in plants: initiation and elaboration of leaves. Trends Genet 31:300-306

Sun Y, Zhou Q, Zhang W, Fu Y, Huang H (2002) ASYMMETRIC LEAVES1, an Arabidopsis gene that is involved in the control of cell differentiation in leaves. Planta 214:694-702
Tamura K, Stecher G, Peterson D, Filipski A, Kumar S (2013) MEGA6: molecular evolutionary genetics analysis version 6.0. Mol Biol Evol 30:2725-2729

Uchida N, Kimura S, Koenig D, Sinha N (2010) Coordination of leaf development via regulation of KNOX1 genes. J Plant Res 123:7-14

Vlad D, Kierzkowski D, Rast MI et al (2014) Leaf shape evolution through duplication, regulatory diversification, and loss of a homeobox gene. Science 343:780-783

Xu Y, Sun Y, Liang W, Huang H (2002) The Arabidopsis AS2 gene encoding a predicted leucine-zipper protein is required for the leaf polarity formation. Acta Bot Sin 44:1194-1202

Yue S, Zhang W, Li FL, Guo YL, Liu TL, Huang H (2000) Identification and genetic mapping of four novel genes that regulate leaf development in Arabidopsis. Cell Res 10:325-335

Zhang Z, Wood WI (2003) A profile hidden Markov model for signal peptides generated by HMMER. Bioinformatics 19:307-308

Zhang T, Hu Y, Jiang W et al (2015) Sequencing of allotetraploid cotton (Gossypium hirsutum L. acc. TM-1) provides a resource for fiber improvement. Nat Biotechnol 33:531-537

Zhu QH, Zhang J, Liu D, Stiller W, Liu D, Zhang Z, Llewellyn D, Wilson I (2016) Integrated mapping and characterization of the gene underlying the okra leaf trait in Gossypium hirsutum L. J Exp Bot 67:763-774

Publisher's Note Springer Nature remains neutral with regard to jurisdictional claims in published maps and institutional affiliations. 